\title{
Prevalence, risk factors, activity limitation and health care utilization of an obese population-based sample with chronic obstructive pulmonary disease
}

\author{
Nicholas T Vozoris MHSc MD FRCPC ${ }^{1}$, Denis E O'Donnell MD FRCPC FCCP²
}

NT Vozoris, DE O’Donnell. Prevalence, risk factors, activity limitation, and health care utilization of an obese population-based sample with chronic obstructive pulmonary disease. Can Respir J 2012;19(3):e18-e24.

OBJECTIVE: To estimate the prevalence and determine the risk factors and health associations among individuals with combined chronic obstructive pulmonary disease and obesity.

METHODS: Canadian national health survey data from 1994 to 2007 $(n=650,000)$ were used. The presence of COPD was based on health professional-diagnosed self-report. The presence of obesity, defined by body mass index $\geq 30 \mathrm{~kg} / \mathrm{m}^{2}$, was identified using self-reported and measured height and weight. Hospitalization, homecare use, physical activity assessments and socioeconomic data were all self-reported.

RESULTS: In 2005, the prevalence of obesity in COPD $(n=3470)$ and non-COPD $(n=92,237)$ individuals was $24.6 \%$ and $17.1 \%$, respectively $(\mathrm{P}<0.0001)$. In contrast to the non-COPD group, in which obesity prevalence increased by $38 \%$ over 14 years, obesity prevalence increased by only $5 \%$ in people with COPD over this same time period. Female sex was the only independent risk factor for obesity in COPD. Previous smoking, residing in Atlantic Canada and the Territories, and low education level were independent risk factors for obesity in the non-COPD group, but not in the COPD group. The odds of physical activity limitation and health care utilization were significantly higher among obese individuals with COPD compared with nonobese COPD and obese non-COPD groups.

CONCLUSIONS: The prevalence of obesity was higher in COPD, and exceeded that of the larger non-COPD group throughout the 13-year observation period. The presence of obesity in COPD was associated with significantly higher risk of severe activity limitation and increased health care utilization. The combination of obesity and COPD has major implications for health care delivery that has not been previously appreciated.

Key Words: COPD; Epidemiology; Health; Obesity; Physical activity

\author{
La prévalence, les facteurs de risque, les limites à \\ l'activité et l'utilisation des soins de santé d'un \\ échantillon en population de personnes obèses atteintes \\ d'une maladie pulmonaire obstructive chronique
}

\begin{abstract}
OBJECTIF : Évaluer la prévalence et déterminer les facteurs de risque et les associations sur la santé des personnes présentant une maladie pulmonaire obstructive chronique (MPOC) associée à l'obésité.

MÉTHODOLOGIE : Les chercheurs ont utilisé les données tirées d'enquêtes canadiennes sur la santé menées entre 1994 et 2007 ( $n=650$ 000). Ils ont déterminé la présence de MPOC d'après une autoévaluation découlant d'un diagnostic posé par un professionnel de la santé. Ils ont établi la présence d'obésité, définie par un indice de masse corporelle égal ou supérieur à $30 \mathrm{~kg} / \mathrm{m}^{2}$, au moyen de l'autoévaluation ou de la mesure du poids et de la taille. L'hospitalisation, le recours à des soins à domicile, les évaluations d'activité physique et les données socioéconomiques étaient tous autoévalués.
\end{abstract}

RÉSULTATS : En 2005, la prévalence d'obésité chez les personnes atteintes d'une MPOC $(n=3470)$ ou non $(n=92237)$ s'élevait à $24,6 \%$ et à $17,1 \%$, respectivement $(\mathrm{P}<0,0001)$. Contrairement au groupe sans MPOC, chez qui la prévalence d'obésité avait augmenté de $38 \%$ sur une période de 14 ans, la prévalence d'obésité n'avait augmenté que de $5 \%$ au cours de la même période chez les personnes atteintes d'une MPOC. Le sexe féminin était le seul facteur de risque indépendant d'obésité en cas de MPOC. Le tabagisme antérieur, le fait d'habiter dans la région atlantique et dans les territoires du Canada et un faible niveau de scolarité étaient des facteurs de risque indépendants d'obésité dans le groupe sans MPOC, mais pas dans celui ayant une MPOC. Le risque de limite à l'activité physique et de recours aux soins de santé était considérablement plus élevé chez les personnes obèses ayant une MPOC que chez les personnes non obèses ayant une MPOC ou sans MPOC.

CONCLUSIONS : La prévalence d'obésité était plus élevée chez les personnes ayant une MPOC et dépassait celle du groupe plus important sans MPOC au cours de la période d'observation de 13 ans. La présence d'obésité en cas de MPOC s'associait à un risque considérablement plus élevé de grave limite à l'exercice et d'augmentation du recours aux soins de santé. L'association d'obésité et de MPOC a des conséquences majeures sur la prestation des soins de santé, lesquelles n'ont pas été évaluées auparavant.

care utilization. The specific objectives of the present study were to: estimate prevalence and 13-year trends of obesity in patients with COPD compared with non-COPD; identify socioeconomic risk factors for obesity in COPD compared with non-COPD; examine associations between physical activity levels among individuals with combined obesity and COPD compared with nonobese COPD and non-COPD obese; to determine whether health care utilization is increased in obese COPD compared with nonobese COPD and non-COPD obese individuals. with more severe COPD (6-8), but extreme obesity in COPD has also been associated with increased respiratory-related mortality (9). Obese subjects with COPD demonstrate reduced exercise capacity as measured by 6 min walk distance $(5,10)$, but not as measured by cycle endurance tests $(11-12)$. To begin to reconcile these apparent contradictions, a better understanding of the interactions among COPD, obesity, activity limitation and health care utilization is needed.

Access to a large, multiyear nationally representative population sample provided a unique opportunity to explore these complex interrelationships among COPD, obesity, physical inactivity and health

\section{METHODS}

Data sources

The present study was conducted using data from Canada's national health surveys: the National Population Health Survey (NPHS) and the Canadian Community Health Survey (CCHS), the latter of which suptaken by Statistics Canada to collect cross-sectional, self-reported planted the NPHS in 2000. The NPHS and the CCHS were under-

\footnotetext{
${ }^{1}$ Division of Respirology, Department of Medicine, St Michael's Hospital, Toronto; Division of Internal Medicine, Department of Medicine,

Kingston General Hospital and Queen's University; 'Division of Respiratory and Critical Care Medicine, Department of Medicine,

Queen's University and Kingston General Hospital, Kingston, Ontario

Correspondence: Dr Nicholas T Vozoris, Division of Respirology, St Michael's Hospital, 30 Bond Street, Toronto, Ontario M5B 1 W8.

Telephone 416-864-6026, fax 416-864-5649, e-mail nick.vozoris@utoronto.ca
} 


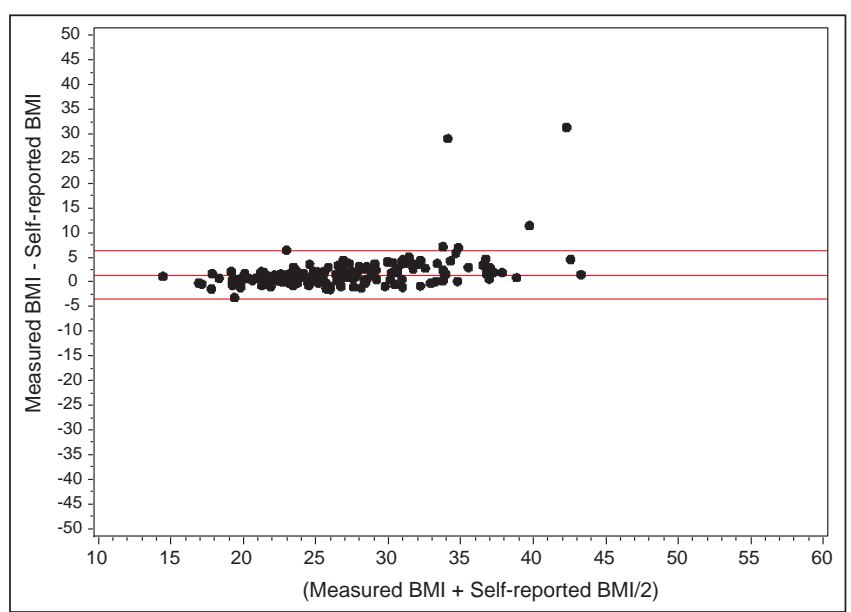

Figure 1) Bland-Altman plot of agreement between measured and selfreported body mass index (BMI) among 2005 Canadian Community Health Survey subsample responders with chronic obstructive pulmonary disease. Horizontal lines show the mean difference $\left(+1.28 \mathrm{~kg} / \mathrm{m}^{2}\right)$ and $95 \%$ limits of agreement $\left(-3.96 \mathrm{~kg} / \mathrm{m}^{2},+6.52 \mathrm{~kg} / \mathrm{m}^{2}\right)$ between measured and self-reported BMI

sociodemographic and health data every two years on a nationally representative sample of $98 \%$ of the Canadian population $\geq 12$ years of age. A description of the survey designs and methodologies appears elsewhere (13-14). Study design and participant recruitment were identical between the NPHS and CCHS, with the exception that the CCHS collected data for much larger sample sizes than the NPHS to allow for analysis at the subprovincial level. Three cycles of the NPHS were completed (1994, 1996 and 1998) before the CCHS began to be administered (2000/2001, 2003, 2005 and 2007). See Appendix 1 for the sample sizes and overall response rates for these surveys.

\section{COPD definition}

All NPHS and CCHS cycles collected information on COPD in the same way, based on health professional-diagnosed, self-reported chronic bronchitis, emphysema and COPD among individuals $\geq 30$ years of age (in the 2005 sample, 25\% [3273 individuals] were $<30$ years of age), with the medical condition lasting, or expected to last, for six months or longer. For the purposes of the present study, respondents were categorized as having COPD if they reported affirmatively to receiving a health professional diagnosis of either chronic bronchitis or emphysema or COPD, and if they were $\geq 40$ years of age. Individuals 30 to 39 years of age self-reporting health professional-diagnosed COPD in the NPHS/CCHS (in the 2005 sample, 410 individuals [2.0\% of individuals] 30 to 39 years of age) were categorized as not having COPD in the present study because it was believed that these young individuals represented misclassified cases of COPD. Individuals who declined to state or did not know their COPD status were excluded from the study sample (approximately $0.1 \%$ in the 2005 CCHS sample). CCHS selfreport of health professional diagnosis for several chronic diseases have been found to have fair to good levels of agreement with populationbased health administrative disease case ascertainment (15). The increasing prevalence of COPD according to age in the sample (Appendix 2), the extremely small percentage of individuals $<40$ years of age self-reporting health professional-diagnosed COPD $(2.0 \%$ in 2005 ) and the high prevalence of current or former smoking in COPD (83\% in 2005), all support the likelihood of accurate diagnosis of COPD by health-professional diagnosed self-report. The definition of COPD was not limited to only current and former smokers because it is increasingly recognized that there are other contributors to the development of COPD beyond active cigarette smoking, including passive smoke exposure (16-17) and occupational exposures (17).

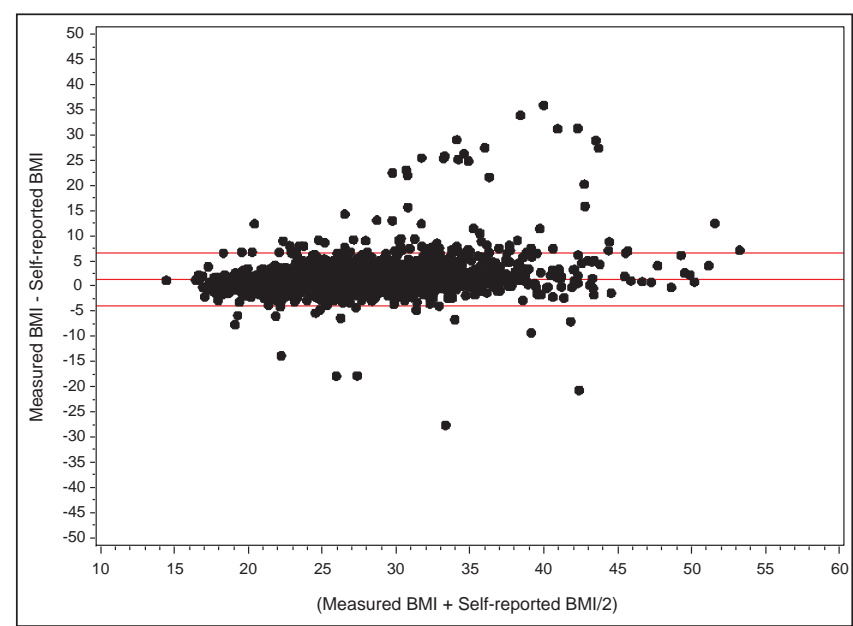

Figure 2) Bland-Altman plot of agreement between measured and selfreported body masss index among 2005 Canadian Community Health Survey subsample responders without chronic obstructive pulmonary disease. Horizontal lines show the mean difference $\left(+1.38 \mathrm{~kg} / \mathrm{m}^{2}\right)$ and $95 \%$ limits of agreement $\left(-3.50 \mathrm{~kg} / \mathrm{m}^{2},+6.26 \mathrm{~kg} / \mathrm{m}^{2}\right)$ between measured and self-reported body mass index

Validation of self-reported body mass index

All NPHS and CCHS cycles collected information on body mass index (BMI) based on self-reported height and weight. The 2005 CCHS cycle also collected information on BMI based on objectively measured height and weight for a randomly selected subsample of survey participants while maintaining proportional representation from all regions of the country $(n=4735,54 \%$ female). Measuring of height and weight followed a standard protocol using a digital weight scale and measuring tape (18). BMI classifications were as follows: underweight $<18.5 \mathrm{~kg} / \mathrm{m}^{2}$; normal weight $18.5 \mathrm{~kg} / \mathrm{m}^{2}$ to $24.9 \mathrm{~kg} / \mathrm{m}^{2}$; overweight $25 \mathrm{~kg} / \mathrm{m}^{2}$ to $29.9 \mathrm{~kg} / \mathrm{m}^{2}$; obese $30 \mathrm{~kg} / \mathrm{m}^{2}$ to $34.9 \mathrm{~kg} / \mathrm{m}^{2}$; and morbid obesity $>35 \mathrm{~kg} / \mathrm{m}^{2}$, following WHO cut-off values (19). Respondents from whom height and weight were not obtained were excluded from the study sample (for the 2005 CCHS, self-reported BMI was not obtained on $3.8 \%$ of the entire sample; measured BMI was not obtained on $1.9 \%$ of the selected subsample). Bland-Altman plots of agreement between measured and self-reported BMI using the CCHS 2005 data showed that mean differences between measured and self-reported BMI in the COPD and non-COPD groups were minimal and of limited clinical significance: $+1.38 \mathrm{~kg} / \mathrm{m}^{2}$ (95\% limits of agreement $\left.=-3.50 \mathrm{~kg} / \mathrm{m}^{2},+6.26 \mathrm{~kg} / \mathrm{m}^{2}\right)$ for COPD; $+1.28 \mathrm{~kg} / \mathrm{m}^{2}(95 \%$ limits of agreement $=-3.96 \mathrm{~kg} / \mathrm{m}^{2},+6.52 \mathrm{~kg} / \mathrm{m}^{2}$ ) for non-COPD (Figures 1 and 2). The proportion of misclassification of individuals with obesity and morbid obesity when using self-reported BMI versus measured BMI was also small: $6.5 \%$ among COPD, $11.6 \%$ among non-COPD (Table 1). These results enabled confident use of self-reported BMI data, which were available for all survey cycles, to investigate trends in obesity in COPD over time.

\section{Physical activity assessment}

Respondents were classed as physically inactive if their total daily energy expenditure was $<1.5 \mathrm{kcal} / \mathrm{kg} / \mathrm{day}$, based on Canadian government standards $(18,20)$ (see Appendix 3 for further details regarding derivation of this variable). Respondents were classed as sedentary if they reported they "usually sit during the day and don't walk around very much". Respondents were classed as having restricted activity if they sometimes or often had difficulty with simple activities such as walking, climbing stairs and bending. Nonresponse to the physical activity assessment questions was very low $(0.2 \%$ to $2.5 \%$, depending on the variable). 
TABLE 1

Proportion of misclassification of individuals with obesity and morbid obesity when using self-reported body mass index (BMI) versus measured BMI using data from the the 2005 Canadian Community Health Survey

\begin{tabular}{|c|c|c|c|c|c|c|c|}
\hline & & \multicolumn{2}{|c|}{ COPD } & & & \multicolumn{2}{|c|}{ Non-COPD } \\
\hline & & \multicolumn{2}{|c|}{ Measured BMI } & & & \multicolumn{2}{|c|}{ Measured BMI } \\
\hline & & Nonobese & Obese & & & Nonobese & Obese \\
\hline \multirow[t]{2}{*}{ Self-reported BMI } & Nonobese & 69.7 & 6.0 & Self-reported BMI & Nonobese & 80.0 & 10.5 \\
\hline & Obese & 0.5 & 23.8 & & Obese & 1.1 & 17.4 \\
\hline
\end{tabular}

Data presented as \%. COPD Chronic obstructive pulmonary disease

\section{Healthcare utilization assessment}

Survey participants were asked whether they were an overnight patient in a hospital, nursing home or convalescent home in the past 12 months. Information on the reason for being an overnight patient was not collected. Survey participants were also asked about receipt of homecare in the past 12 months paid for by governmental and/or nongovernmental sources. Nonresponse to the health care utilization assessment questions was very low $(<0.1 \%$ for both questions).

\section{Final study sample}

The final study sample for each CCHS cycle consisted of individuals who were $\geq 30$ years of age who responded to questions regarding COPD and self-reported BMI. For example, for the 2005 CCHS, of the 132,221 individuals in the total sample, 95,707 were $\geq 30$ years of age and gave responses to questions on COPD and self-reported BMI. Individuals from the study sample who did not respond to questions on physical activity and health care utilization were not included in these respective health analyses.

\section{Statistical analysis}

All analyses were performed using SAS version 9.1.3 (SAS Institute, USA). The frequencies of self-reported BMI designations were calculated for individuals with COPD and without COPD using CCHS 2005 data. Frequencies of self-reported obese (BMI $\geq 30 \mathrm{~kg} / \mathrm{m}^{2}$ ) designations in the COPD and non-COPD populations were calculated for the years 1994 to 2007. Although data from the 2005 CCHS were not the most recent survey data available, they were used for analyses of risk factors, activity limitation and health care utilization of obesity in COPD because the data were validated against objective BMI measurement. Use of self-reported rather than objectively measured BMI data from the 2005 CCHS were selected in the analyses of risk factors, activity limitation and health care utilization of obesity in COPD, because the former variable had data on a much larger number of individuals $(132,221$ versus 4735$)$.

Multiple logistic regression was used to identify independent sociodemographic risk factors for obesity in both the COPD and non-COPD groups. Descriptive analysis and multiple logistic regression were used to examine the health outcomes. Comparisons of obese individuals with COPD versus nonobese individuals with COPD, obese individuals without COPD and nonobese individuals without COPD were undertaken to tease out whether any observed differences in health outcomes were due to BMI designation, COPD designation or both. Two multiple logistic regression models were run for each health outcome variable. The first model included all sociodemographic factors listed in Table 2. The second model included all variables in the first model, plus health-professional diagnosed self-reported heart disease, hypertension and diabetes. The purpose of this second regression model was to investigate whether any observed health outcome differences were independent of these comorbid cardiovascular diseases, which individuals with COPD are also at increased risk (21-22).

The CCHS uses a complex sampling design, using stratification and multistage clustering. To account for the unequal probabilities of selecting respondents, all results were weighted using the survey sample weights provided. All sample weights were rescaled before each analysis by dividing the original weight by the average weight of respondents included in the specific analysis, according to Statistics Canada guidelines (23). To account for the effects of stratification and clustering on variance estimates, all confidence intervals were calculated using bootstrap resampling techniques with bootstrap weights created by Statistics Canada. Ethics approval was granted by the University of Toronto (Toronto, ONtario) Office of Research Ethics.

\section{RESULTS}

Of the 95,707 individuals $\geq 30$ years of age who responsed to questions on COPD and self-reported BMI in the 2005 CCHS, 3470 (3.6\%) had COPD. Among individuals with COPD, 853 (24.6\%) self-reported obesity and $298(8.6 \%)$ self-reported morbid obesity. Significantly greater numbers of individuals with COPD compared with individuals without COPD had designations of underweight, obesity and morbid obesity, and significantly fewer had designations of normal weight and overweight, based on self-reported BMI (Figure 3).

Between 1994 and 2007, the prevalence of self-reported COPD increased from $1.5 \%$ to $3.8 \%$ (a $153 \%$ increase) (Figure 4). During this same time period, the prevalence of self-reported obesity increased from $13.3 \%$ to $18.5 \%$ (a $38 \%$ increase) in the non-COPD population. While the prevalence of self-reported obesity among individuals with COPD has always been higher compared with the non-COPD population, the change between 1994 and 2007 was minimal (25.6\% to $27 \%$ [5\% increase]).

Frequencies of sociodemographic factors among obese and nonobese individuals with COPD and without COPD are presented in Table 2. Multiple logistic regression analysis showed that sociodemographic risk factors associated with obesity in the non-COPD population, such as low education level and former smoking, were not risk factors for obesity in the COPD group. While the odds of obesity among residents of Atlantic Canada and the Territories were $>1.00$ for both individuals with COPD and without COPD, results were not statistically significant for individuals with COPD. Some socioeconomic factors were similarly protective for obesity in non-COPD and COPD (ie, increasing age, being an immigrant, current smoking). Other socioeconomic variables were protective in non-COPD but not in COPD (ie, residing in Quebec), and while the odds of obesity among visible minorities and among individuals with high income and education levels were $<1.00$ for both individuals with COPD and without COPD, results were not statistically significant for individuals with COPD. Increasing age was more strongly inversely related with obesity in COPD versus nonCOPD. In contrast to the non-COPD population in which females were protected from obesity, women were independently associated with obesity in the COPD group (Table 2).

Frequencies of activity limitation and health care utilization measures and among obese individuals with COPD, nonobese individuals with COPD, obese individuals without COPD, and nonobese individuals without COPD are presented in Figure 5. After controlling for sociodemographic confounders, obese individuals with COPD compared with nonobese individuals with COPD, obese individuals without COPD, and nonobese individuals without COPD had significantly increased odds of being physically inactive, being sedentary and having restricted activity level (Table 3 ). These results remained significant after additionally controlling for cardiovascular comorbidities, with the exception of physical inactivity among obese individuals with COPD compared with non-COPD obese.

After controlling for sociodemographic confounders, obese individuals with COPD compared with nonobese individuals with COPD, obese individuals without COPD and nonobese individuals without 
TABLE 2

Proportions of demographic characteristics among obese individuals and nonobese individuals with chronic obstructive pulmonary disease (COPD) and without COPD, plus adjusted odds ratios for obesity among individuals with COPD and without COPD, using the 2005 Canadian Community Health Survey

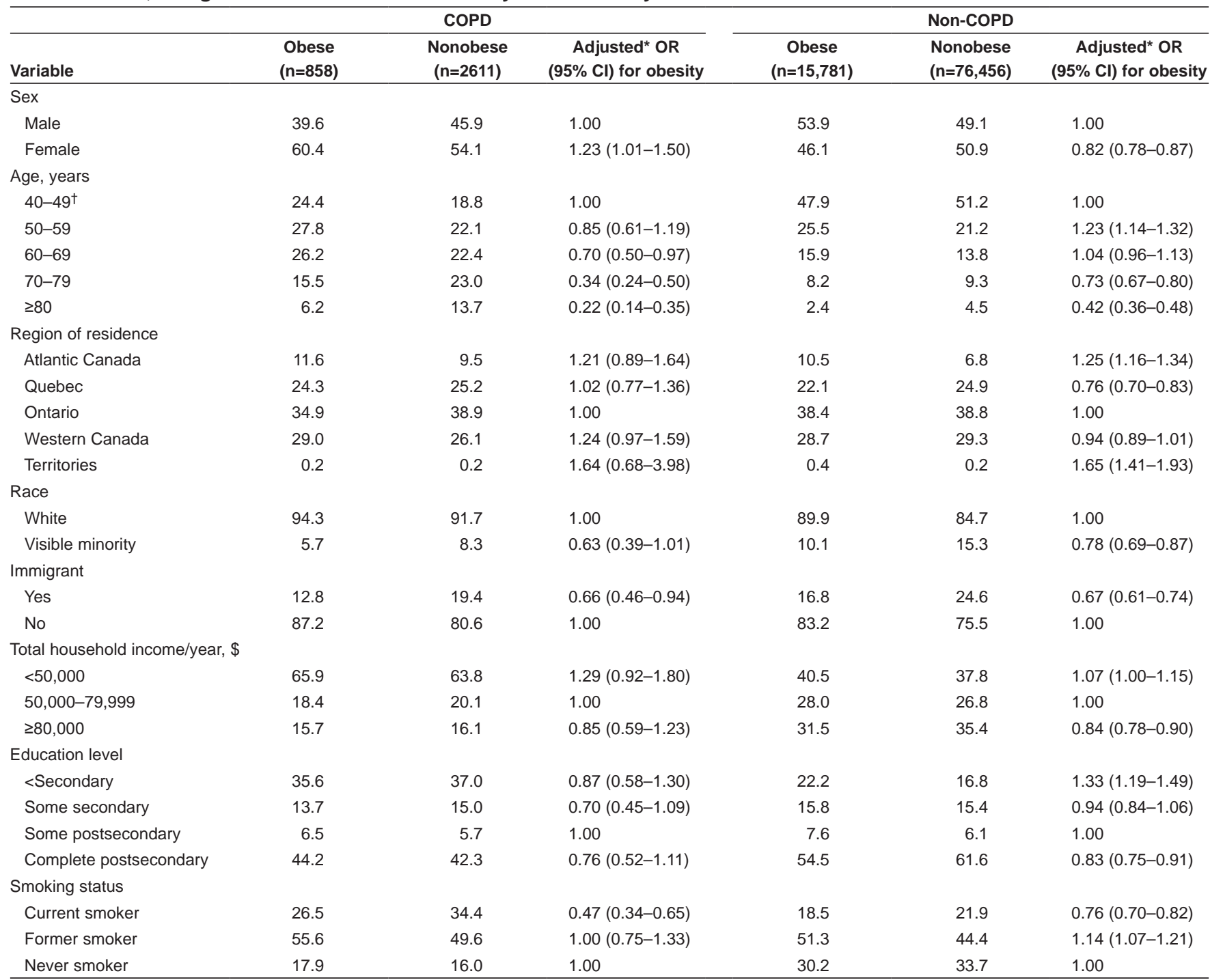

Data presented as \% unless otherwise indicated. ${ }^{*}$ Regression equation included all of the variables listed; ${ }^{\dagger}$ This reference age group includes 30 - to 49 -year-olds for non-COPD only

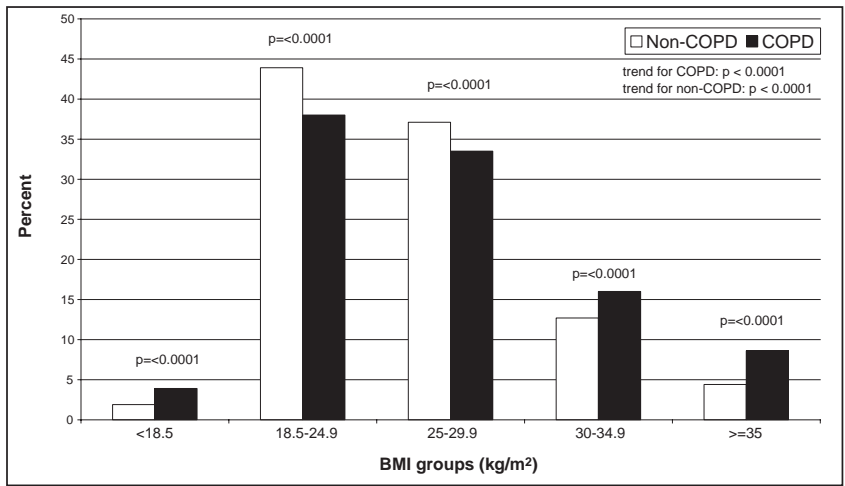

Figure 3) Frequency of individuals with chronic obstructive pulmonary disease (COPD) $(n=3470)$ and without COPD $(n=92,237)$ across body mass index (BMI) groups, based on self-reported height and weight in the total 2005 sample

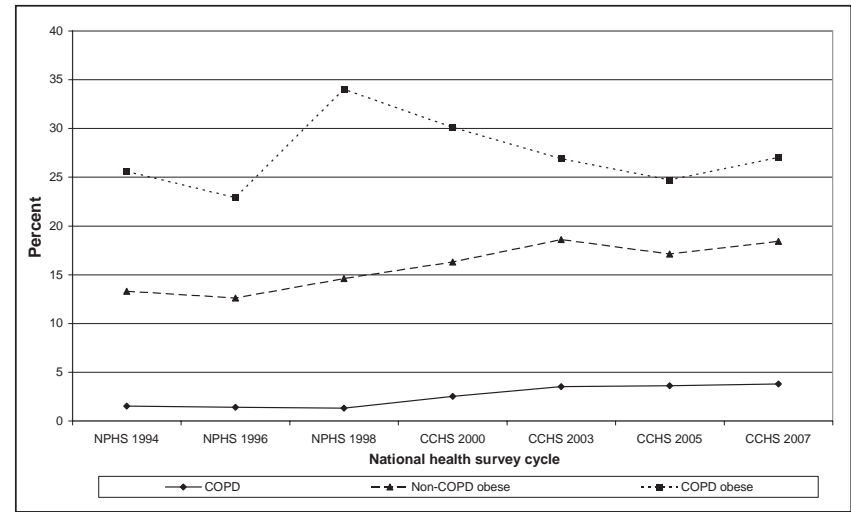

Figure 4) Changes in the prevalences of overall chronic obstructive pulmonary disease (COPD) and obesity (body mass index $>30 \mathrm{~kg} / \mathrm{m}^{2}$ ) among individuals with COPD and without COPD, between 1994 and 2007 in Canada. CCHS Canadian Community Health Survey; NPHS National Population Health Survey 


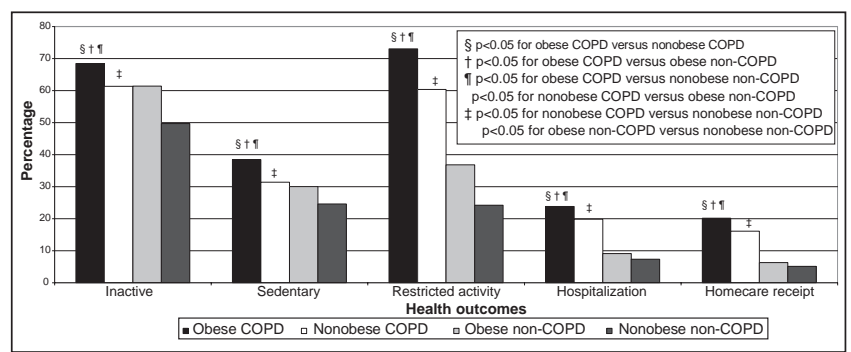

Figure 5) Frequencies of activity limitation and health care utilization among obese individuals with chronic obstructive pulmonary disease (COPD), nonobese individuals with COPD, obese individuals without COPD, and nonobese individuals without COPD in the 2005 Canadian Community Health Survey

COPD, had significantly increased odds of being an overnight patient and receiving homecare (Table 3 ). These results remained significant after additionally controlling for cardiovascular comorbidities, with the exception of being an overnight patient among obese individuals with COPD compared with nonobese COPD.

\section{DISCUSSION}

The present study generated several novel findings. First, the prevalences of obesity and morbid obesity in health professional-diagnosed self-reported COPD were significantly higher than in the non-COPD population. Second, in contrast to the non-COPD population, in which obesity steadily escalated over 13 years, obesity remained relatively stable among individuals with COPD. Third, predisposing and protective factors for obesity were different between the obese COPD and obese non-COPD groups. Finally, increased and clinically significant physical activity limitation and health care utilization were observed among obese individuals with COPD compared with nonobese COPD and non-COPD obese individuals.

The present study was the first to examine inter-relationships among COPD, obesity, activity limitation and health care utilization using a large, nationally-representative population sample with data spanning a 13-year time period. Although self-reported BMI was used for much of our analysis, this was found to have good agreement with objectively measured BMI in both the COPD and non-COPD populations in subanalysis validation. Response rates to questions on COPD, BMI and health outcomes were excellent. Eleven sociodemographic and health status variables (ie, sex, age, region of residence, race, immigration status, income level, education level, smoking status, hypertension, diabetes and heart disease) were included in our logistic regression models of health outcomes to control for potential confounding factors.

Previous studies have reported a higher prevalence of obesity $(4,5)$ among individuals with COPD compared with individuals without COPD, but these estimates were based on data from relatively small local populations. Our Canadian prevalence estimate of obesity in COPD (25\%) is similar to South American estimates (23\%) (24), higher than that reported in the Dutch population (18\%) (4) and much lower than that reported in a small cohort in northern California (USA) (54\%) (5).

Recent epidemiological studies have confirmed a steady and dramatic increase in the prevalence of obesity throughout the western world (1). The lack of significant overall change in the prevalence of obesity in COPD between 1994 and 2007 compared with the nonCOPD population is noteworthy and suggests that COPD is itself a risk factor for obesity. However, the substantial rise in the prevalence of obesity in the COPD group in 1998, followed by its gradual decline to pre-1998 levels, is curious and unexplained.

\section{TABLE 3}

Odds of physical activity limitation and health care utilization in the past 12 months among obese individuals with chronic obstructive pulmonary disease (COPD) versus nonobese COPD, obese non-COPD and nonobese non-COPD, using data from the 2005 Canadian Community Health Survey

\begin{tabular}{|c|c|c|c|}
\hline & \multicolumn{3}{|c|}{ Physical activity measures } \\
\hline & & Adjusted OR Model $1^{\dagger}(95 \% \mathrm{Cl})$ & Adjusted OR Model $2^{\ddagger}(95 \% \mathrm{Cl})$ \\
\hline & Inactive*, n & of inactivity* & of inactivity \\
\hline Obese COPD versus nonobese COPD & 834 versus 2074 & $1.51(1.22-1.87)$ & $1.42(1.14-1.77)$ \\
\hline Obese COPD versus obese non-COPD & 834 versus 10,481 & $1.14(0.94-1.38)$ & $1.09(0.89-1.32)$ \\
\hline \multirow[t]{2}{*}{ Obese COPD versus nonobese non-COPD } & 834 versus 36,131 & $1.95(1.62-2.34)$ & $1.92(1.59-2.32)$ \\
\hline & $\begin{array}{l}\text { Reporting being sedentary } \\
\text { during the day, } \mathbf{n}\end{array}$ & $\begin{array}{l}\text { Adjusted* OR Model } 1^{\dagger}(95 \% \mathrm{Cl}) \\
\text { of reporting being sedentary }\end{array}$ & $\begin{array}{c}\text { Adjusted OR Model } 2^{\ddagger}(95 \% \mathrm{Cl}) \text { of } \\
\text { reporting being sedentary }\end{array}$ \\
\hline Obese COPD versus nonobese COPD & 460 versus 1037 & $1.49(1.21-1.84)$ & $1.31(1.05-1.63)$ \\
\hline Obese COPD versus obese non-COPD & 460 versus 4935 & $1.36(1.13-1.63)$ & $1.31(1.09-1.58)$ \\
\hline \multirow[t]{2}{*}{ Obese COPD versus nonobese non-COPD } & 460 versus 16,512 & $2.15(1.79-2.57)$ & $1.91(1.59-2.29)$ \\
\hline & Reported activity restriction, $\mathbf{n}$ & $\begin{array}{l}\text { Adjusted* OR Model } 1^{\dagger}(95 \% \mathrm{Cl}) \\
\text { of reporting activity restriction }\end{array}$ & $\begin{array}{l}\text { Adjusted OR Model } 2^{\ddagger}(95 \% \mathrm{Cl}) \text { of } \\
\text { reporting activity restriction }\end{array}$ \\
\hline Obese COPD versus nonobese COPD & 863 versus 2178 & $2.05(1.62-2.58)$ & $1.99(1.56-2.54)$ \\
\hline Obese COPD versus obese non-COPD & 863 versus 6946 & $3.39(2.77-4.15)$ & $3.16(2.56-3.90)$ \\
\hline \multirow[t]{3}{*}{ Obese COPD versus nonobese non-COPD } & 863 versus 20,860 & $6.55(5.40-7.94)$ & $5.90(4.81-7.24)$ \\
\hline & \multicolumn{3}{|c|}{ Health care utilization measures } \\
\hline & $\begin{array}{c}\text { Were an } \\
\text { overnight patient, } \mathrm{n}\end{array}$ & $\begin{array}{c}\text { Adjusted OR Model } 1^{\dagger}(95 \% \mathrm{Cl}) \\
\text { of being an overnight patient }\end{array}$ & $\begin{array}{c}\text { Adjusted OR Model } 2^{\ddagger}(95 \% \mathrm{Cl}) \text { of } \\
\text { being an overnight patient }\end{array}$ \\
\hline Obese COPD versus nonobese COPD & 277 versus 759 & $1.42(1.10-1.82)$ & $1.16(0.88-1.51)$ \\
\hline Obese COPD versus obese non-COPD & 277 versus 1817 & $2.59(2.07-3.24)$ & $2.10(1.67-2.63)$ \\
\hline \multirow[t]{3}{*}{ Obese COPD versus nonobese non-COPD } & 277 versus 6581 & $3.32(2.70-4.08)$ & $2.27(1.83-2.83)$ \\
\hline & & Adjusted OR Model $1^{\dagger}(95 \% \mathrm{Cl})$ & Adjusted OR Model $2^{\ddagger}(95 \% \mathrm{Cl})$ \\
\hline & Used homecare, n & of using homecare & of using homecare \\
\hline Obese COPD versus nonobese COPD & 257 versus 702 & $1.72(1.33-2.23)$ & $1.50(1.14-1.97)$ \\
\hline Obese COPD versus obese non-COPD & 257 versus 1384 & $2.46(1.95-3.10)$ & $2.13(1.67-2.71)$ \\
\hline Obese COPD versus nonobese non-COPD & 257 versus 5264 & $3.36(2.70-4.17)$ & $2.59(2.06-3.25)$ \\
\hline
\end{tabular}

*Inactivity defined as a total daily energy expenditure value of <1.5 kcal/kg/day, as per Canadian Fitness and Lifestyle Research Institute cut-offs; ${ }^{\dagger}$ Model 1 adjusts for sex, age, region of residence, race, immigration status, income level, education level and smoking status; ${ }^{\ddagger}$ Model 2 adjusts for all the variables in Model 1 , plus health professional-diagnosed heart disease, health professional-diagnosed hypertension and health professional-diagnosed diabetes 
Similar to previous reports $(25,26)$, we identified several predisposing factors (male sex, former smoking and low education level) and protective factors (being a visible minority) for obesity in the general population. However, these predisposing and protective factors were not associated with obesity in the COPD group, suggesting that determinants of obesity are, in part, different in COPD and non-COPD populations. The discrepancy in predisposing and protective factors for obesity in COPD and non-COPD may, in part, be related to small sample sizes, especially in the COPD group. Our finding that female sex predisposes to obesity in COPD, but is protective in those without COPD, is striking. Sex discrepancies relating to obesity in COPD have been previously reported. A greater proportion of obesity was found among women (56\%) versus men $(44 \%)$ in a South American population sample with COPD (24), and a higher mean BMI was reported among women $\left(24.1 \mathrm{~kg} / \mathrm{m}^{2}\right)$ versus men $\left(22.5 \mathrm{~kg} / \mathrm{m}^{2}\right)$ in a study of Dutch individuals with COPD (7).

One potential explanation for the observed COPD-obesity relationship is activity avoidance due to COPD-related dyspnea. Potentially in keeping with this, we have shown substantially increased (216\% higher) levels of severe activity restriction (involving limitation in such basic physical activities as walking and bending) among obese individuals with COPD compared with obese individuals without COPD. The fact that we found physical activity limitation among obese individuals with COPD that significantly exceeded that in both the nonobese COPD and nonCOPD obese groups, suggests that COPD and obesity each independently contribute to the reduced activity level in obese COPD. Factors that likely contribute to increased dyspnea during weight-bearing exercise in obese COPD include the relatively increased metabolic and ventilatory demands of locomotion compared with normal weight COPD. The contributory role of restrictive ventilatory mechanics in exertional dyspnea causation in obese COPD patients appears to be less important than previously postulated (11). Activity avoidance as a result of COPD-related dyspnea may also explain why female sex was found to predispose to obesity in COPD in the present study. Greater degrees of dyspnea $(27,28)$ and activity limitation (28) have been previously reported among women with COPD versus men with COPD, even when matched for the same forced expiratory volume in $1 \mathrm{~s}(29,30)$, which potentially predisposes women with COPD to gain more weight than their male counterparts.

While previous population studies have proposed a possible protective health effect of obesity in COPD (6-8), we found substantially increased health care utilization among obese individuals with COPD. Significant differences in hospitalization between obese COPD and nonobese COPD disappeared when cardiovascular comorbidities were accounted for, suggesting that these latter factors were contributory to the increased healthcare utilization in obese COPD. The reduced mortality among obese individuals with COPD in the study by Landbo et al (7) was exclusively limited to individuals with severe COPD, suggesting that obesity may exert differing effects in COPD depending on disease severity. More recent population-level data have shown significantly increased respiratory-related, but not all-cause, mortality among individuals with COPD with extreme obesity (BMI $\left.\geq 40 \mathrm{~kg} / \mathrm{m}^{2}\right)$ (9).

\section{Limitations}

Although the present study was based on data spanning over a 10 -year time period, it was cross-sectional in nature and potential causal links cannot be established. The presence of COPD, cardiovascular comorbidities, activity limitation and health care utilization, were based on self-report, thereby potentially introducing recall bias. These same variables may have also been underestimated because of social desirability bias. There must also be some degree of misclassification between individuals categorized with and without COPD, and with and without cardiovascular comorbities, because classifications relied purely on health professional-diagnosed self-report, and no objective confirmation of diagnoses (eg, with spirometry) was available. Studies of small and selected populations with physician-diagnosed COPD have found COPD overdiagnosis ranging from 5\% to $31 \%(31-33)$, and COPD underdiagnosis ranging from $68 \%$ to $81 \%(31,34)$, when spirometry was undertaken for diagnosis confirmation. The substantially higher percentage of COPD underdiagnosis versus COPD overdiagnosis associated with physician-diagnosed COPD suggests that our findings of increased activity limitation and health care utilization among obese COPD versus obese non-COPD and nonobese non-COPD may, in fact, be underestimates. Although the $17 \%$ of individuals categorized with COPD who were neither current nor former smokers may, in part represent misclassification, we did not exclude never-smokers from our COPD definition because other contributors to the development of COPD, such as passive cigarette smoke exposure (16-17) and occupational exposures (17), are recognized beyond active cigarette smoking. Although our analysis controlled for a large number of sociodemographic and cardiovascular confounders, we had no means by which to adjust our results for COPD severity. Other 'harder' clinical outcomes, such as exacerbation rate and mortality, were also not available. Longitudinal data, with objectively confirmed COPD and comorbidities, would be needed to confirm the study findings.

In conclusion, the prevalence of obesity among individuals with health professional-diagnosed self-reported COPD in Canada is high $(25 \%)$, greater than in the non-COPD population and has remained relatively stable over a 13 -year observation period. Predisposing and protective factors for obesity were different in the COPD and nonCOPD groups. Severe physical activity limitation and increased health care utilization were found among obese individuals with health professional diagnosed self-reported COPD compared with nonobese individuals with COPD and non-COPD obese individuals. Further study with longitudinal data and spirometrically-confirmed COPD would be required to confirm these findings. The novel finding of increased and clinically important activity restriction and health care utilization among obese individuals with COPD compared with nonobese COPD subjects may have important implications for future management. It remains to be seen whether targeted weight reduction interventions and exercise training programs can ameliorate increased morbidity in individuals in whom obesity and COPD are combined.

DISCLOSURE: Denis O'Donnell has served on advisory boards for Boehringer Ingelheim, GSK, Novartis, Nycomed and Pfizer; has received lecture fees from Astra Zeneca, Boehringer Ingelheim, GSK and Pfizer; and has received industry-sponsored grants from Astra Zeneca, Boehringer Ingelheim, GSK, Merck Frosst Canada, Novartis and Pfizer.

APPENDIX 1

Canadian national health survey cycle total samples sizes and combined household-person level response rates

\begin{tabular}{lcc}
\hline $\begin{array}{l}\text { National health survey } \\
\text { cycle, year }\end{array}$ & Total sample size, $\mathbf{n}$ & $\begin{array}{c}\text { Household-person level } \\
\text { response rate, \% }\end{array}$ \\
\hline NPHS, 1994 & 17,626 & 88.7 \\
NPHS, 1996 & 81,804 & 82.6 \\
NPHS, 1998 & 17,244 & 87.6 \\
CCHS, 2000/2001 & 130,880 & 84.7 \\
CCHS, 2003 & 134,072 & 80.7 \\
CCHS, 2005 & 132,947 & 78.9 \\
CCHS, 2007 & 131,061 & 77.6 \\
\hline
\end{tabular}

CCHS Canadian Community Health Survey; NPHS National Population Health Survey

APPENDIX 2

Prevalence of health-professional diagnosed self-reported chronic obstructive pulmonary disease (COPD) according to age cateogory using the 2005 Canadian Community Health Survey database

\begin{tabular}{lc}
\hline Age cateogory, years & Prevalence of COPD, \% \\
\hline $40-49$ & 2.7 \\
$50-59$ & 3.9 \\
$60-69$ & 5.8 \\
$70-79$ & 8.1 \\
$\geq 80$ & 9.7
\end{tabular}




\section{APPENDIX 3 \\ Description of physical activity variable}

The CCHS asked participants about the frequency and average duration of 21 different types of physical activities (see list below). The daily energy expenditure $(E E)$ was calculated for each physical activity by multiplying the reported frequency (over 12 months), average duration (in hours), and the associated metabolic energy cost (MET value) for each activity expressed as kilocalories expended per kilogram of body weight per hour of activity and dividing by 365 . MET values are available for different activity intensity levels: low, medium, and high intensity. Information on activity intensity level was not collected by the CCHS, and low intensity MET values were adopted for each activity for the survey. MET values were obtained from the Canadian Fitness and Lifestyle Research Institute $(18,20)$. The total daily EE level for each respondent was calculated by adding together the daily $E E$ for each reported physical activity participated in. Questionnaire-based estimates of EE like that in the CCHS are frequently used (35), and have been found to correlate well with energy expenditure estimates based on pedometer output (36) and doubly-labeled water methods (37-39)

Sports and physical activities included in the $\mathrm{CCHS}$ from which total daily energy expenditure was calculated include: baseball or softball, basketball, bicycling, bowling, dance, exercise class or aerobics, fishing, gardening or yard work, golfing, home exercise, ice hockey, ice skating, in-line skating or rollerblading, jogging or running, skiing or snowboarding, soccer, swimming, tennis, volleyball, walking, weight training and other (up to 3 could be listed).

\section{REFERENCES}

1. Finucane MM, Stevens GA, Cowan MJ, et al. National, regional, and global trends in body-mass index since 1980: Systematic analysis of health examination surveys and epidemiological studies with 960 country-years and 9.1 million participants. Lancet 2011;377:557-67.

2. Buist AS, McBurnie MA, Vollmer WM, et al. International variation in the prevalence of COPD (the BOLD Study): A population-based prevalence study. Lancet 2007;370:741-50.

3. Guerra S, Sherill DL, Bobadilla A, Martinez FD, Barbee RA. The relation of body mass index to asthma, chronic bronchitis, and emphysema. Chest 2002;122:1256-63.

4. Steuten LMG, Cruetzberg EC, Vrijhoef HJM, Wouters EF. COPD as a multicomponent disease: Inventory of dyspnea, underweight, obesity and fat free mass depletion in primary care. Prim Care Resp J 2006;15:84-91.

5. Eisner MD, Blanc PD, Sidney S, et al. Body composition and functional limitation in COPD. Respir Res 2007;8:7-17.

6. Chailleux E, Laaban JP, Veale D. Prognostic value of nutritional depletion in patients with COPD treated by long-term oxygen therapy: Data from the ANTADIR observatory. Chest 2003;123:1460-6.

7. Landbo C, Prescott E, Lange P, Vestbo J, Almdal TP. Prognostic value of nutritional status in chronic obstructive pulmonary disease. Am J Respir Crit Care Med 1999;160:1856-61.

8. Wilson DO, Rogers RM, Wright EC, Anthonisen NR. Body weight in chronic obstructive pulmonary disease. The National Institutes of Health Intermittent Positive Pressure Breathing Trial. Am Rev Respir Dis 1989;139:1435-8.

9. Jordan JG, Mann JR. Obesity and mortality in persons with obstructive lung disease using data from NHANES III. Southern Med J 2010;103:323-30.

10. Sava F, Laviolette L, Bernard S, Breton MJ, Bourbeau J, Maltais F. The impact of obesity on walking and cycling performance and response to pulmonary rehabilitation in COPD. BMC Pulm Med 2010;10:55.

11. Ora J, Laveneziana P, Ofir D, Deesomchok A, Webb KA, O'Donnell DE. Combined effects of obesity and chronic obstructive pulomary disease on dyspnea and exercise tolerance. Am J Resp Crit Care Med 2009;180:964-71.

12. Laviolette L, Sava F, O'Donnell DE, et al. Effect of obesity on constant workrate exercise in hyperinflated men with COPD. BMC Pulm Med 2010;102:613-9.

13. Beland Y. Canadian Community Health Survey - methodological overview. Health Rep 2002;13:9-14.

14. Tambay JL, Catlin G. Sample design of the National Population Health Survey. Health Rep 1995;7:29-38.
15. Lix LM, Yogendran MS, Shaw SY, Burchill C, Metge T, Bond R. Population-based data sources for chronic disease surveillance. Chronic Diseases in Canada 2008;29:31-8.

16. Yin P, Jiang CQ, Cheng KK, et al. Passive smoking exposure and risk of COPD among adults in China: The Guangzhou Biobank Cohort Study. Lancet 2007;370:751-7.

17. Salvi SS, Barnes PJ. Chronic obstructive pulmonary disease in nonsmokers. Lancet 2009;374:733-43.

18. Statistics Canada, Health Statistics Division. Canadian Community Health Survey Cycle 3.1 (2005), Public Use Microdata File, Integrated Derived Variable and Grouped Variable Specifications. Ottawa: Statistics Canada, 2006.

19. World Health Organization. Global Database on Body Mass Index. BMI Classification. < http://apps.who.int/bmi/index.jsp > (Accessed May 3, 2012).

20. Cameron C, Wolfe K, Craig CL. Physical activity and sport: Encouraging children to be active. Ottawa: Canadian Fitness and Lifestyle Research Institute, 2007.

21. Curkendall SM, DeLuise C, Jones JK, et al. Cardiovascular disease in patients with chronic obstructive pulmonary disease, Saskatchewan, Canada. Ann Epidemiol 2006;16:63-70.

22. Huiart L, Ernst P, Suissa S. Cardiovascular morbidity and mortality in COPD. Chest 2005;128:2640-6.

23. Statistics Canada, Health Statistics Division. Canadian Community Health Survey (CCHS) Cycle 3.1 2005, User Guide for the Public Use Microdata File (PUMF). Ottawa: Statistics Canada, 2006.

24. Montes de Oca M, Tálamo C, Perez-Padilla R, et al. Chronic obstructive pulmonary disease and body mass index in five Latin America cities: The PLATINO study. Respir Med 2008;102:642-50.

25. Macdonald SM, Reeder BA, Chen Y, Després JP. Obesity in Canada: A descriptive analysis. Canadian Heart Health Surveys Research Group. CMAJ 1997;157(Suppl 1):S3-9.

26. Ostbye T, Pomerleau J, Speechley M, Pederson LL, Speechley KN. Correlates of body mass index in the 1990 Ontario Health Survey. CMAJ 1995;152:1811-7.

27. de Torres JP, Casanova C, Hernández C, et al. Gender associated differences in determinants of quality of life in patients with COPD: A case series study. Health Qual Life Outcomes 2006;4:72.

28. Osman IM, Godden DJ, Friend JA, Legge JS, Douglas JG. Quality of life and hospital re-admission in patients with chronic obstructive pulmonary disease. Thorax 1997;52:67-71.

29. de Torres JP, Casanova C, Hernández C, Abreu J, Aguirre-Jaime A, Celli BR. Gender and COPD in patients attending a pulmonary clinic. Chest 2005;128:2012-6.

30. Guenette JA, Jensen D, Webb KA, Ofir D, Raghavan N, O'Donnell DE. Sex differences in exertional dyspnea in patients with mild COPD: Physiological mechanisms. Respir Physiol Neurobiol 2011;177:218-27.

31. Hill K, Goldstein RS, Guyatt GH, et al. Prevalence and underdiagnosis of chronic obstructive pulmonary disease among patients at risk in primary care. CMAJ 2010;182:673-8.

32. Jones RC, Dickson-Spillmann M, Mather MJ, Marks D, Shackell BS. Accuracy of diagnostic registers and management of chronic obstructive pulmonary disease: The Devon primary care audit. Respir Res 2008;9:62-71.

33. Walters JA, Walters EH, Nelson M, et al. Factors associated with misdiagnosis of COPD in primary care. Prim Care Respir J 2011;doi: 10.4104/pcrj.2011.00039.

34. Bednarek M, Maciejewski J, Wozniak M, Kuca P, Zielinski J. Prevalence, severity and underdiagnosis of COPD in the primary care setting. Thorax 2008;63:402-7.

35. Neilson HK, Robson PJ, Friedenreich CM, Csizmadi I. Estimating activity energy expenditure: How valid are physical activity questionnaires? Am J Clin Nutr 2008;87:279-91.

36. Edelman B, Smits G. The pedomter: A reassessment of its usefullness in the measurement of activity level. Percept Mot Skills 1984;58:151-8.

37. Goran MT, Potchman ET. Total energy expenditure and energy requirements in healthy elderly persons. Metabolism 1992;41:744-53.

38. Phillipaerts RM, Westerterp KR, Lefevre J. Doubly labelled water validation of three physical activity questionnaires. Int J Sports Med 1999; 20:284-9.

39. Stuten LK, Turen DL, Howell WH, et al. Validation of the Arizona Acitivity Frequency Questionnaire using doubly labelled water. Med Sci Sports Exerc 2001;33:1959-67. 


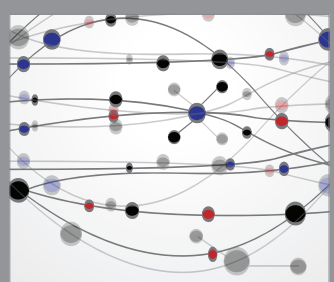

The Scientific World Journal
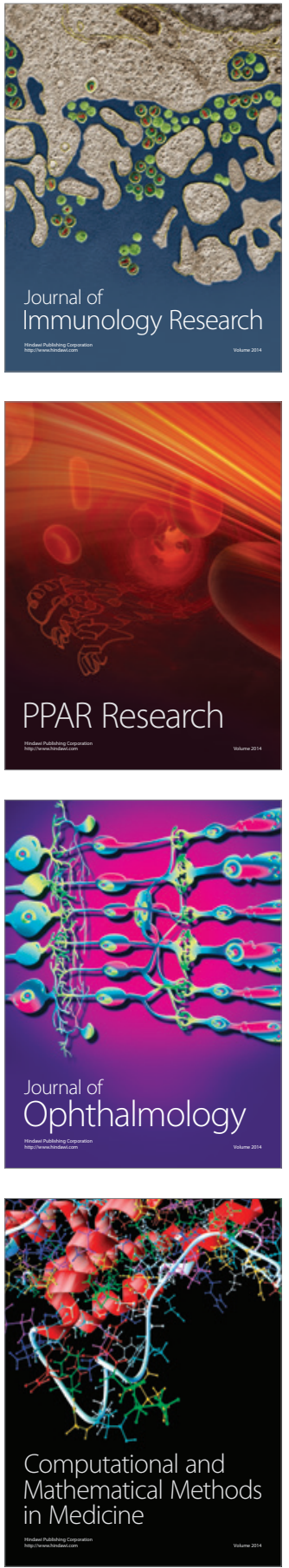

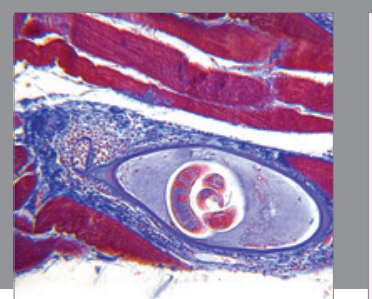

Gastroenterology Research and Practice

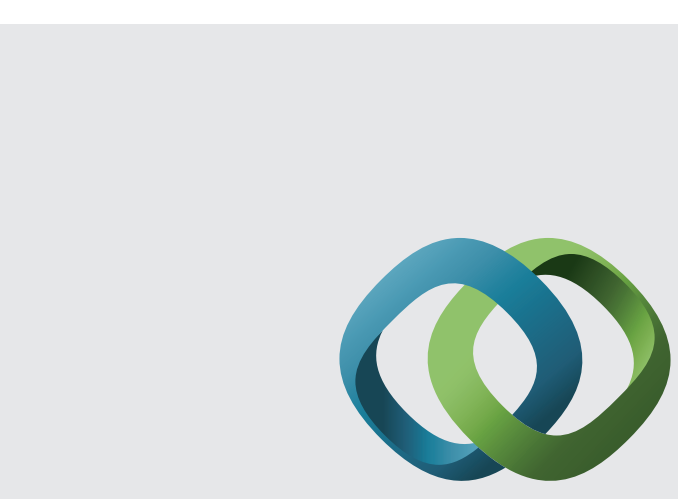

\section{Hindawi}

Submit your manuscripts at

http://www.hindawi.com
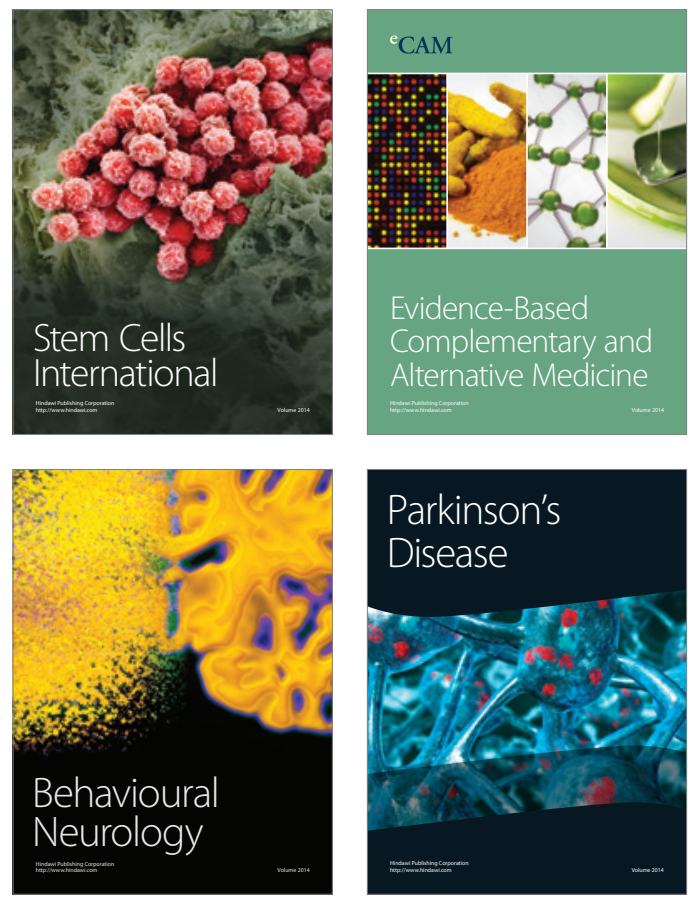
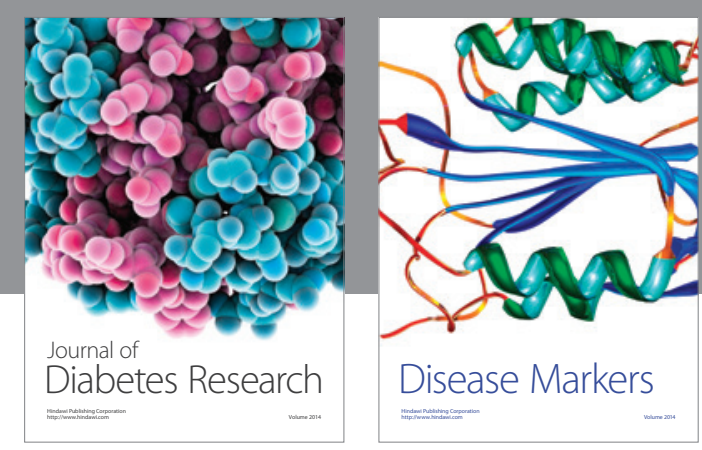

Disease Markers
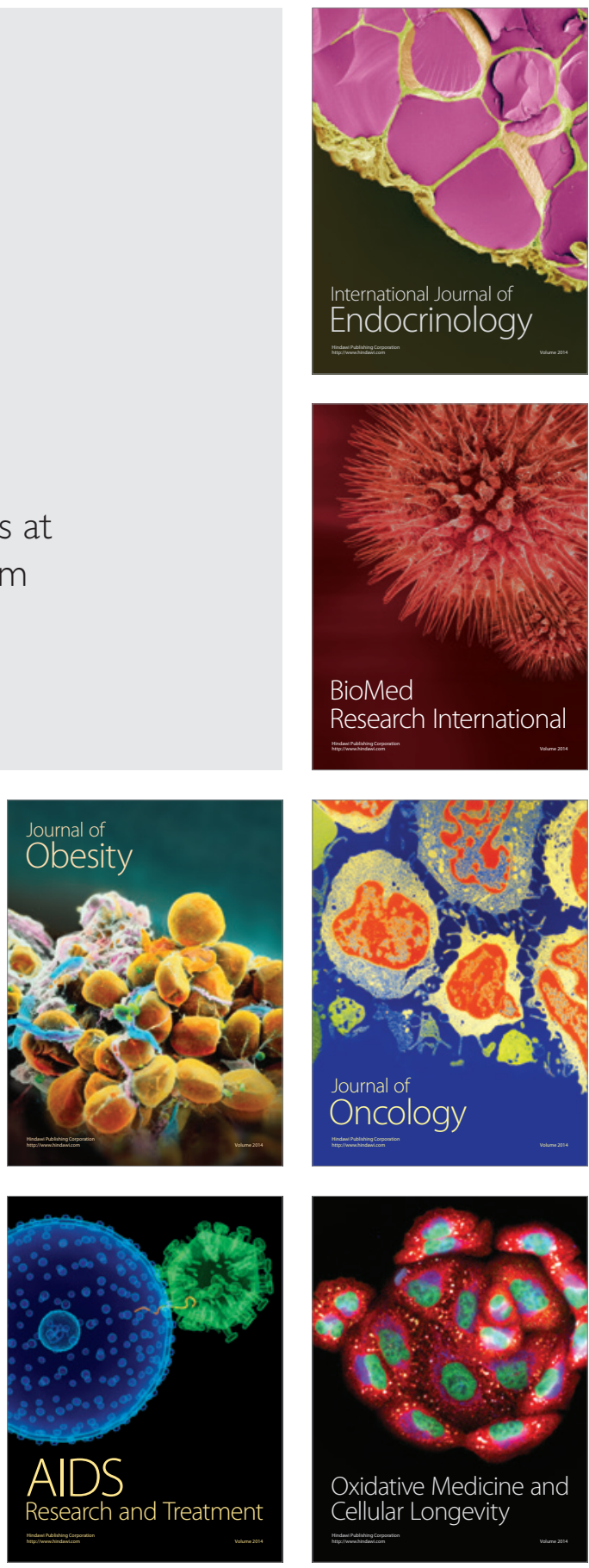